\title{
Análisis evolutivo de la calidad de la solución final de diálisis en España y Portugal
}

\author{
Mercedes Serrano Arias \\ Ana Rodríguez Rebollar \\ Ángeles González Carcedo \\ Natalia Carrillo López \\ José Luis Fernández Martín \\ Jorge B Cannata Andía
}

\author{
Servicio de Metabolismo Óseo y Mineral \\ Instituto "Reina Sofía" de Investigación \\ Hospital Universitario Central de Asturias \\ Universidad de Oviedo
}

\begin{abstract}
RESUMEN
Las soluciones de diálisis contaminadas con aluminio representan la fuente de exposición al aluminio más peligrosa en pacientes en diálisis. El objetivo de este trabajo fue conocer la concentración de aluminio en la solución final de diálisis de los centros españoles y portugueses en el año 2004, y comparar estos resultados con los obtenidos en estudios previos.
\end{abstract}

En el presente estudio los centros españoles participantes fueron 248 y los portugueses 54 . El aluminio pudo ser medido en todas las muestras recibidas. Estudios similares y realizados con la misma metodología fueron hechos en España en 1990, 1994 y 1999, y en Portugal en 1999 y 2001.

El porcentaje de centros españoles con aluminio en la solución final de diálisis inferior a $2 \mu \mathrm{g} / \mathrm{L}$ se ha ido incrementando progresivamente desde el primer estudio en 1990, hasta este último de 2004. En Portugal, el porcentaje de centros con aluminio en la solución final de diálisis inferior de $2 \mu \mathrm{g} / \mathrm{L}$ fue de $91,7 \%$ en $1999,85,7 \%$ en 2001 y de $94,4 \%$ en 2004.

En todos los estudios realizados en España el porcentaje de centros con niveles indetectables de aluminio $(\leq 1 \mu \mathrm{g} / \mathrm{L})$ aumentó; esta mejoría se observó hasta 1999, ya que en

\footnotetext{
Correspondencia:

Mercedes Serrano Arias

Servicio de Metabolismo Óseo y Mineral

Hospital Universitario Central de Asturias

c) Julián Clavería s/n

33006 Oviedo (Asturias)

E-mail:mercedes@hca.es
}

2004 el porcentaje se mantuvo estable. En Portugal dicho porcentaje se ha mantenido estable desde el año 1999 hasta la actualidad.

El estudio realizado en 2004 en España y Portugal y su comparación con estudios similares previos, demuestran que la calidad de las soluciones de diálisis ha mejorado notablemente durante los últimos 15 años.

$\begin{aligned} \text { PALABRAS CLAVE: } & \text { ALUMINIO } \\ & \text { DIÁLISIS } \\ & \text { ESPECTROMETRÍA DE ABSORCIÓN } \\ & \text { ATÓMICA }\end{aligned}$

\section{EVOLUTIONAL ANALYSIS OF THE QUALITY OF THE FINAL DIALYSIS SOLUTION IN SPAIN AND PORTUGAL}

\section{SUMMARY}

Dialysis solutions contaminated with aluminium represent the most dangerous source of exposure to aluminium in dialysis patients. The aim of this work was to determine the concentration of aluminium in the final dialysis solution in Spanish and Portuguese hospitals in 2004, and compare these results to those obtained in previous studies.

In this study, the number of Spanish hospitals participating was 248 and number of Portuguese was 54. The aluminium could be measured in all the samples received. Similar studies using the same methodology were carried out in Spain in 1990, 1994 and 1999, and in Portugal in 1999 and 2001. 
The percentage of Spanish centres with less than $2 \mu \mathrm{g} / \mathrm{L}$ of aluminium in the final dialysis solution has increased gradually since the first study in 1990, until this study in 2004 . In Portugal, the percentage of hospitals with less than 2 $\mu \mathrm{g} / \mathrm{L}$ of aluminium in the final dialysis solution was $91.7 \%$ in $1999,85.7 \%$ in 2001 and $94.4 \%$ in 2004.

In all the studies carried out in Spain, the percentage of hospitals with undetectable levels of aluminium $(\leq 1 \mu \mathrm{g} / \mathrm{L})$ increased; this improvement was observed up to 1999 , since in 2004 the percentage remained stable. In Portugal this percentage has remained stable since 1999 until the present.

The study carried out in 2004 in Spain and Portugal and its comparison with previous similar studies shows that the quality of dialysis solutions has improved significantly over the last 15 years.

\section{KEYWORDS ALUMINIUM DIALYSIS \\ ATOMIC ABSORPTION SPECTROMETRY}

\section{INTRODUCCIÓN}

Las dos fuentes principales de aluminio a las que están expuestos los pacientes en diálisis son la solución de diálisis contaminada con aluminio y su ingesta, fundamentalmente el hidróxido de aluminio ${ }^{1,2}$. Sin embargo, son los líquidos de diálisis contaminados, los que representan un mayor riesgo de exposición al aluminio para estos pacientes ${ }^{1,3-7}$, ya que el mismo se incorpora directamente a la sangre sin ninguna barrera que impida su absorción. Entre los años 70 y comienzos de los años 80, se encontró una asociación entre el contenido de aluminio del agua de suministro de la red, usada para la preparación de los líquidos de diálisis, con la encefalopatía dialítica y la osteomalacia fracturante. Dicha asociación hizo que la AAMI (Association for the Advancement of Medical Instrumentation) y un poco más tarde las autoridades de Canadá y Europa, sugirieran un umbral de $10 \mu \mathrm{g} / \mathrm{L}$ de aluminio en el líquido de diálisis como el umbral de seguridad para evitar la neurotoxicidad resultante de la exposición al líquido de diálisis contaminado con aluminio.

En varios trabajos previos $^{4-7}$, se ha insistido sobre la necesidad de controlar y mantener de forma permanente los niveles de aluminio de la solución final de diálisis por debajo de un "umbral eficaz" de seguridad, para evitar la transferencia de aluminio de la solución de diálisis al paciente y facilitar la movilización de aluminio desde el paciente a la solución de diálisis8 y así poder minimizar la toxicidad alumínica.

Con la idea de alcanzar dicho objetivo, en 1990 se planteó la realización de estudios transversales periódicos de los centros de diálisis españoles, recayendo la parte logística del estudio en el personal de enfermería del servicio. En la década siguiente se observó una importante y progresiva mejoría ${ }^{9}$, pero todavía casi un $20 \%$ de los centros no habían alcanzado la cifra "ideal" de $2 \mu \mathrm{g} / \mathrm{L}$ de aluminio en la solución final de diálisis. Estudios similares fueron realizados en Portugal, sin que en este país se observara mejoría en relación a los controles previos.

El presente estudio compara los resultados del último estudio multicéntrico español y portugués realizado en 2004 y evalúa la evolución de la calidad de la solución final de diálisis desde 1990 hasta la actualidad.

\section{MATERIAL Y MÉTODOS}

En los meses de febrero-abril de 2004 se realizó el último estudio sobre la concentración de aluminio en las soluciones de diálisis de los centros de diálisis españoles y portugueses. La sistemática empleada fue la misma que en los otros estudios9, invitándose a participar a los centros de diálisis que aparecían censados en el Anuario Nacional de Nefrología. Tras un exhaustivo cribado, se confirmaron 284 centros españoles activos en la actualidad. El número de centros invitados de Portugal fue de 90, obteniéndose su dirección a través de la Sociedad Portuguesa de Nefrología. A todos se les envió una carta con instrucciones para la recogida, manipulación y envío de las muestras junto con dos tubos de poliestireno cristalino, libres de contaminación por aluminio ${ }^{10,11}$. Las muestras fueron recogidas por duplicado, para tener un tubo de reserva en el caso de que alguna de ellas sufriera alguna incidencia en el envío, o por si surgieran dudas en el resultado.

Tres estudios similares siguiendo la misma metodología, fueron realizados en España en 1990, 1994 y 1999. En el primero participaron 190 centros (82\% de los enviados); en dicho estudio sólo se pudieron analizar las muestras de 160 centros. En 30 casos la determinación no fue posible por tubos rotos o vacíos, o incorrecta identificación de la muestra. En el segundo estudio participaron 205 centros (83,6\%) y fue posible analizar la totalidad de las muestras. En el tercero, realizado en 1999, de los 275 centros invitados, participaron 242 (88\%) y el aluminio pudo ser medido en todas las muestras recibidas. En el último estudio de 2004, de los 284 centros españoles que fueron invitados a participar, respondieron 248 (87\%). El aluminio pudo ser medido en las 248 muestras recibidas.

Los estudios realizados en los centros de diálisis de Portugal en 1999 y 2001 se hicieron con la misma metodología que los españoles. Los centros participantes fueron 60 (67\%) en el 
año 1999 y 41 (46\%) en el 2001 12. En el último estudio de 2004 , los centros portugueses que respondieron fueron 54 , lo que representa el 60\% de los 90 centros que fueron invitados a participar.

En todos los estudios el aluminio se midió por espectrometría de absorción atómica por horno de grafito HGA-600, con un muestreador automático AS-60 y un espectómetro de absorción atómica Z-3030 con corrección de fondo Zeeman de la casa Perkin Elmer, según metodología publicada con anterioridad13. Las determinaciones se realizaron en una habitación libre de partículas (clase 100), para evitar la contaminación de las muestras con polvo atmosférico.

\section{RESULTADOS}

En la figura 1 se muestra la distribución de los centros españoles en los 4 estudios realizados, según el contenido de aluminio en la solución de diálisis. El porcentaje de centros con aluminio inferior a $2 \mu \mathrm{g} / \mathrm{L}$ se ha incrementado progresivamente desde el primer estudio en 1990 (45\%), hasta el último realizado en 2004 (83.4\%).

\section{ESPAÑA}
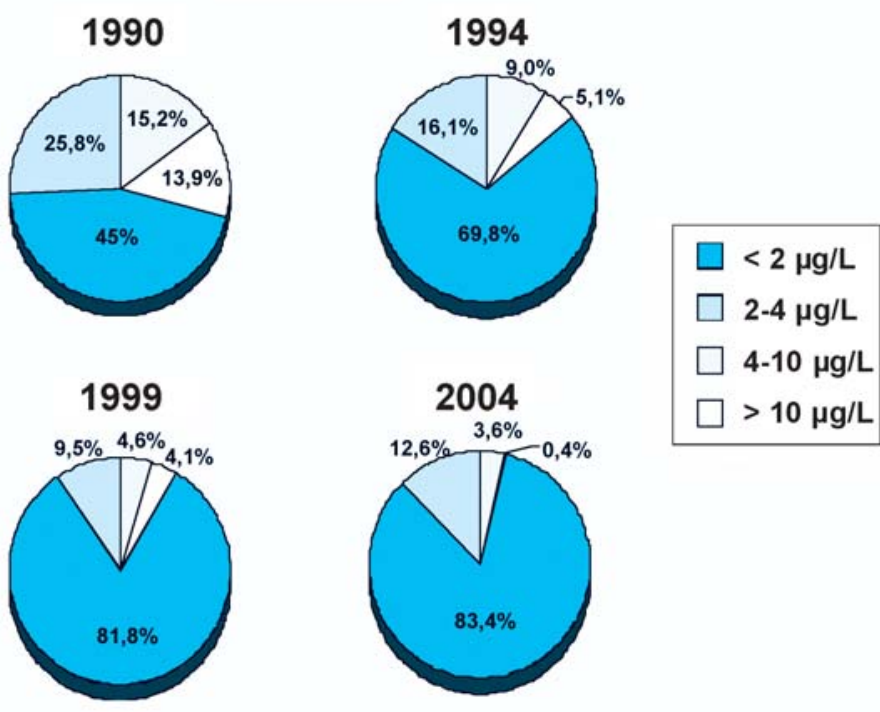

Figura 1. Distribución de los centros según el nivel de aluminio en los tres estudios transversales realizados en España desde 1990 hasta 2004.

El porcentaje de centros con aluminio en la solución final de diálisis de 2-4 $\mu \mathrm{g} / \mathrm{L}$ descendió de $25,8 \%$ en 1990 a 9,5\% en 1999, sin embargo, en 2004 subió a 12,6\% con respecto al anterior (1999). El porcentaje de centros con aluminio en la solución final de diálisis entre 4-10 $\mathrm{\mu g} / \mathrm{L}$ ha disminuido de forma progresiva desde 1990 a 2004. Lo mismo ocurrió con los valores superiores a $10 \mu \mathrm{g} / \mathrm{L}$, que se han reducido de un 13,9\% en 1990, a un 0,4\% en 2004.
La figura 2 muestra los resultados de los centros de diálisis portugueses. El porcentaje de centros con aluminio por debajo de $2 \mu \mathrm{g} / \mathrm{L}$, fue de $91,7 \%$ en 1999 , de $85,7 \%$ en 2001 y de $94,4 \%$ en 2004. El porcentaje de centros con niveles de aluminio en la solución final de diálisis superiores a $10 \mu \mathrm{g} / \mathrm{L}$ fue bajo en 1999 , y no hubo ningún centro con estas características en 2004. En el año 2001 se registraron los peores resultados, con un 7,1\% de los centros con niveles superiores a $10 \mu \mathrm{g} / \mathrm{L}$.

\section{PORTUGAL}

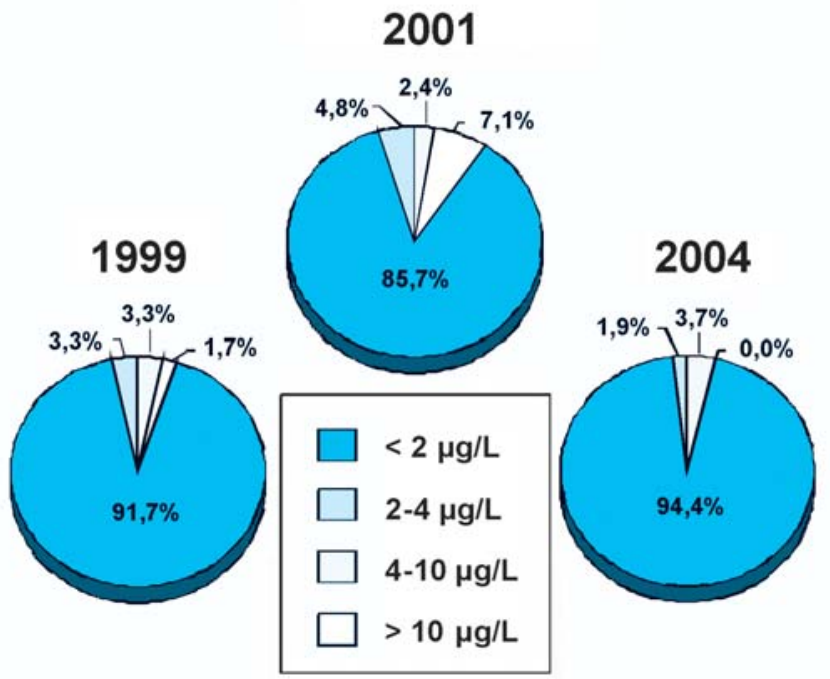

Figura 1. Distribución de los centros según el nivel de aluminio en los tres estudios transversales realizados en España desde 1990 hasta 2004.

En la tabla 1 se muestra la escala completa del porcentaje de centros con concentraciones de aluminio de 1 a $10 \mu \mathrm{g} / \mathrm{L}$ en España y Portugal. En todos los estudios realizados el porcentaje de centros con niveles indetectables de aluminio $(£ 1 \mu \mathrm{g} / \mathrm{L})$ aumentó en España hasta 1999, y en el último corte transversal de 2004 dicho porcentaje se mantuvo estable. En Portugal dicho porcentaje se ha mantenido más o menos estable desde el año 1999 hasta 2004.

\begin{tabular}{|lc|c|c|c|c|c|c|}
\hline & \multicolumn{3}{c}{ España } & \multicolumn{3}{c|}{ Portugal } \\
\hline Al (ug/L) & $\mathbf{1 9 9 0}$ & $\mathbf{1 9 9 4}$ & $\mathbf{1 9 9 9}$ & $\mathbf{2 0 0 4}$ & $\mathbf{1 9 9 9}$ & $\mathbf{2 0 0 1}$ & $\mathbf{2 0 0 4}$ \\
\hline $\mathbf{1}$ & 22.9 & 41.2 & 66.9 & 65.1 & 78.3 & 75.6 & 77.8 \\
\hline $\mathbf{2}$ & 47.1 & 69.8. & 82.6 & 85.4 & 91.7 & 85.4 & 94.4 \\
\hline $\mathbf{3}$ & 62.4 & 80.0 & 87.6. & 92.4 & 95.0 & 87.5 & 94.4 \\
\hline $\mathbf{4}$ & 70.7 & 85.9 & 91.3 & 96.0 & 95.0 & 90.2 & 96.3 \\
\hline $\mathbf{5}$ & 77.1 & 89.0 & 93.0 & 97.7 & 96.7 & 92.7 & 98.1 \\
\hline $\mathbf{6}$ & 80.3 & 89.8 & 94.2 & 98.0 & 98.3 & 92.7 & 98.1 \\
\hline $\mathbf{7}$ & 82.2 & 91.0 & 95.0 & 99.0 & 98.3 & 92.7 & 100.0 \\
\hline $\mathbf{8}$ & 84.1 & 92.2 & 95.0 & 99.0 & 98.3 & 92.7 & 100.0 \\
\hline $\mathbf{9}$ & 85.4 & 94.5 & 95.5 & 99.6 & 98.3 & 92.7 & 100.0 \\
\hline $\mathbf{1 0}$ & 86.0 & 94.9 & 95.9 & 99.6 & 98.3 & 92.7 & 100.0 \\
\hline
\end{tabular}

Tabla 1. Porcentaje de centros con concentraciones de aluminio por debajo de las indicadas en la columna de la izquierda en España y Portugal en los distintos cortes transversales realizados. 
En la figura 3 se muestra la distribución geográfica de los centros españoles participantes en el estudio, junto con el porcentaje de centros con contenidos de aluminio en la solución final de diálisis inferior a $2 \mu \mathrm{g} / \mathrm{L}$. La comunidad con un menor porcentaje de centros con aluminio $<2 \mu \mathrm{g} / \mathrm{L}$ es Canarias $(61,5 \%)$, mientras que en el otro extremo se encuentra Cataluña con un $91,9 \%$ de centros con soluciones finales de diálisis con aluminio $\leq 2 \mu \mathrm{g} / \mathrm{L}$.

Hay otras tres regiones con un $100 \%$ de centros con aluminio $\leq 2 \mu \mathrm{g} / \mathrm{L}$, pero este dato tiene valor limitado dado que en ambos casos han participado solamente 1 ó 2 centros.

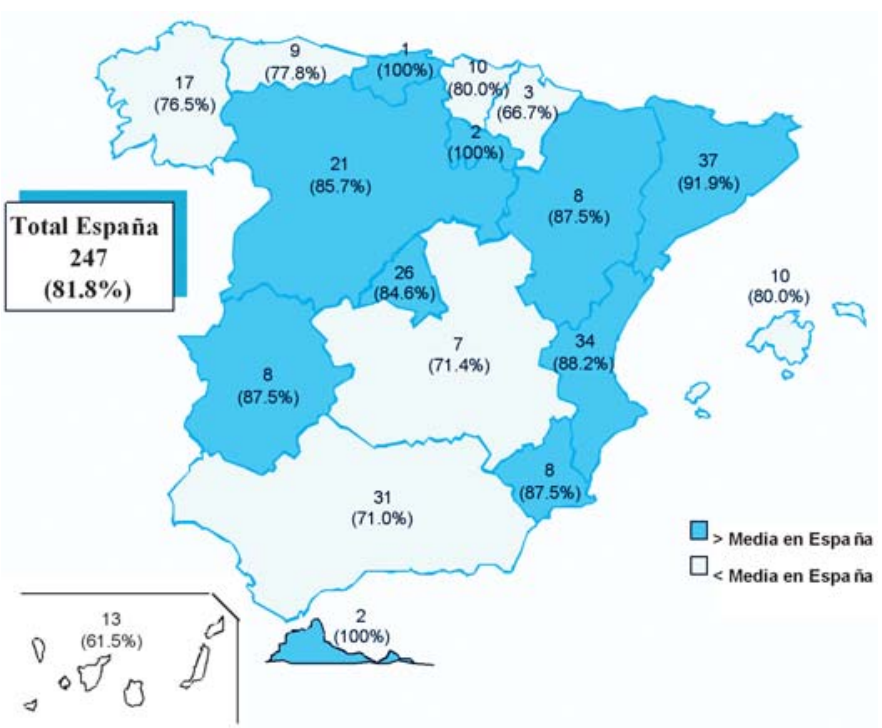

Figura 3. Número de centros de diálisis participantes en cada una de las comunidades autónomas. Entre paréntesis se indica el porcentaje de centros con niveles de aluminio inferior a $2.0 \mathrm{\mu g} / \mathrm{L}$. En color amarillo, las comunidades con un porcentaje de centros con niveles menores de $2.0 \mu \mathrm{g} / \mathrm{L}$ inferior a la media española (81.8\%). En color verde, aquellas comunidades con un porcentaje superior a la media.

\section{DISCUSIÓN}

A lo largo de los últimos quince años se han realizado varios estudios ${ }^{5,8,14}$ encaminados a reducir la exposición al aluminio de los pacientes en diálisis. Ello ha permitido establecer una serie de recomendaciones para minimizar dicha exposición, las cuales estaban basadas en mantener la concentración de aluminio en la solución de diálisis por debajo de un umbral de seguridad $^{4-6,14}$, y en la monitorización frecuente de los mismos $^{8}$. Este nuevo estudio transversal realizado en 2004 nos permite conocer la situación actual de los centros de diálisis españoles, así como la evolución de los mismos desde la realización de los tres estudios transversales previos de 1990, 1994 y 1999. El porcentaje de participación en los cuatro estudios transversales realizados fue siempre superior al $80 \%$, alcan- zando en los dos últimos estudios unos niveles de participación del 88 y $87 \%$ respectivamente, esto hace que los datos obtenidos tengan gran valor dado que pueden ser extrapolables a la totalidad de los centros de diálisis españoles durante un período total de casi 15 años.

El nivel de participación en Portugal no fue tan elevado ni tan homogéneo en los tres estudios realizados (67, 46 y $60 \%$ en 1999, 2001 y 2004 respectivamente). Esta menor participación puede ser debida a los errores en las bases de datos del censo de los centros de diálisis portugueses.

En la figura 1 se puede observar que el porcentaje de los centros españoles con aluminio en la solución de diálisis superior a $10 \mu \mathrm{g} / \mathrm{L}$ era muy elevado en 1990 (13,9\%). Cuatro años más tarde descendió considerablemente hasta un $5,1 \%$ y se mantuvo más o menos estable en el siguiente corte en 1999 $(4,1 \%)$. En el último estudio de 2004 se produjo un marcado descenso, sólo un $0.4 \%$ de los centros presentaron niveles de aluminio en la solución final de diálisis por encima de $10 \mu \mathrm{g} / \mathrm{L}$. El umbral de seguridad de $10 \mu \mathrm{g} / \mathrm{L}$ fue fijado por la Unión Europea en 1986 para evitar la transferencia de aluminio al paciente en diálisis, en una época en la que la exposición al aluminio y los niveles de aluminio sérico de los pacientes era mucho mayor que el observado en la actualidad. Sin embargo, este umbral de seguridad resulta obsoleto, ya que existen estudios que demuestran que en la actualidad las concentraciones de aluminio no deberían superar los $4 \mu \mathrm{g} / \mathrm{L}$, siendo la cifra ideal a alcanzar $2 \mu \mathrm{g} / \mathrm{L}$. Si estos niveles no se logran existe un riesgo de exposición lenta y progresiva al aluminio?

A pesar de que el umbral de seguridad de $10 \mu \mathrm{g} / \mathrm{L}$ está desactualizado, es importante resaltar que en este último estudio de 2004, prácticamente la totalidad de los centros españoles $(99,6 \%)$ (tabla 1) cumplían con ese límite de calidad de las soluciones finales de diálisis.

El umbral de seguridad de concentración de aluminio en las soluciones de diálisis debe adecuarse al nivel medio de aluminio sérico de los pacientes en diálisis y con objeto de permitir un gradiente positivo de aluminio desde el paciente a la solución de diálisis. Por ese motivo, hace 15 años, nuestra primera recomendación fue la de mantener el aluminio en la solución de diálisis por debajo de $5 \mu \mathrm{g} / \mathrm{L} 4,7$, pero a medida que la exposición al aluminio ha ido disminuyendo, los niveles séricos de aluminio de los pacientes han ido descendiendo de forma paralela, hecho que ha motivado que en los últimos años se recomendase un umbral de seguridad de $2-4 \mu \mathrm{g} / \mathrm{L} 5,8$.

El porcentaje de centros españoles con concentraciones de aluminio en la solución final de diálisis inferiores de $2 \mu \mathrm{g} / \mathrm{L}$ ha aumentado progresivamente desde el primer control de 1990 
(45 \%) hasta 2004 (83,4\%), demostrando una importante mejoría en la calidad de los líquidos de diálisis. Este nivel de aluminio en la solución final de diálisis fue el más adecuado cuando en España la concentración media de aluminio sérico era superior a $30 \mu \mathrm{g} / \mathrm{L}$. No obstante, los niveles de aluminio sérico en los pacientes en diálisis en España ha ido descendiendo paulatinamente8, en el año 1996 la media de aluminio sérico estaba en torno a $25 \mu \mathrm{g} / \mathrm{L}$ y en la actualidad se sitúa por debajo de $20 \mu \mathrm{g} / \mathrm{L}$, probablemente cercano a $10 \mu \mathrm{g} / \mathrm{L}$, que es la cifra que durante mucho tiempo ha sido considerada como nivel máximo de aluminio en sujetos con función renal normal.

Si bien no existen evidencias que demuestren que una cifra de aluminio sérico inferior a $20 \mu \mathrm{g} / \mathrm{L}$ se asocie con enfermedad atribuible al aluminio, el objetivo es conseguir que los pacientes de diálisis tengan valores de aluminio sérico lo más cercanos a la población normal; esto se consigue con una concentración de aluminio en la solución final de diálisis $<1 \mu \mathrm{g} / \mathrm{L}$.

Dado que sólo el 10\% de aluminio sérico es dializable $(1 \mu \mathrm{g} / \mathrm{L}$ para un $\mathrm{Al}$ sérico de $10 \mu \mathrm{g} / \mathrm{L}$, valor próximo a la normalidad), la concentración de aluminio en la solución final de diálisis debería ser inferior a esta cifra. En la presentación de los datos hasta $1999^{9}$, se había propuesta la cifra de $<1 \mu \mathrm{g} / \mathrm{L}$ como objetivo a conseguir, para evitar la transferencia de aluminio al paciente.

A lo largo de casi 15 años se ha observado un incremento importante de los centros con aluminio en la solución de diálisis por debajo de este umbral, $1 \mu \mathrm{g} / \mathrm{L}$, límite de detección de la técnica de determinación de aluminio. Los centros con solución final de diálisis de excelente calidad $(<1 \mu \mathrm{g} / \mathrm{L})$ han ido mejorando progresivamente desde un $22,9 \%$ en $1990,41,2 \%$ en $1994,66,9 \%$ en 1999 y se ha estabilizado $(65,1 \%)$ en 2004. La mejora que se produjo entre 1990 y 1994 es razonable y fácil de explicar ya que en 1990 no todos los centros disponían de ósmosis inversa para el tratamiento del agua. Sin embargo, las mejoras observadas a partir de 1994 no se deben fundamentalmente a mejoras tecnológicas, sino probablemente, al mejor mantenimiento y/o control de los sistemas de ósmosis en los centros de diálisis, dado que la mayor parte de las inversiones económicas en sistemas de tratamiento de agua realizadas en España se hicieron antes del segundo estudio.

A pesar del incremento del porcentaje de centros con niveles indetectables de aluminio, este objetivo parece difícil de mejorar, ya que en 1999 el 66,9\% de los centros españoles cumplían con dicho objetivo y en 2004, este porcentaje se ha mantenido, pero no ha podido ser superado.

En una encuesta publicada en $1996^{14}$ en la que participaron el $44 \%$ los centros de diálisis de España, se observó que la ma- yoría de centros sólo cuantificaba la concentración de aluminio en la solución de diálisis 1 ó 2 veces por año. Desde entonces, se ha insistido ${ }^{8}$ en que una determinación frecuente de dichos niveles (mensual), es una política de prevención segura y muy barata para evitar sobre todo exposiciones masivas accidentales al aluminio, pero también exposiciones sutiles y mantenidas a dicho elemento. El correcto mantenimiento del sistema de ósmosis y un control rutinario frecuente del mismo es, sin duda, la estrategia adecuada a seguir para alcanzar el objetivo de mantener las soluciones finales de diálisis con concentraciones de aluminio inferiores a $1 \mu \mathrm{g} / \mathrm{L}$.

La figura 3 muestra la distribución geográfica de los centros participantes en el estudio de 2004. En el estudio global de 2004, el 81,8\% de los centros españoles alcanzaron una concentración de aluminio en la solución final de diálisis inferior a $2 \mu \mathrm{g} / \mathrm{L}$. Muy por debajo del porcentaje global de España se situó Canarias. Tal vez el empleo de desaladoras y de sistemas de potabilización del agua del mar tenga alguna influencia sobre la eficiencia de los sistemas de ósmosis inversa de las unidades de diálisis. En el otro extremo se encuentra Cataluña, en donde 34 de los 37 centros participantes (91,9\%) mostraron niveles de aluminio en la solución final de diálisis por debajo de $2 \mu \mathrm{g} / \mathrm{L}$. Los centros de la Comunidad Valenciana también estuvieron a un nivel similar (88,2\%). También hubo otras comunidades con el 100\% de centros con la solución de aluminio inferior a $2 \mu \mathrm{g} / \mathrm{L}$ (Cantabria, La Rioja, Ceuta y Melilla) pero en ellos se registró un escaso número de centros (1 ó 2).

El hecho de poseer sistemas de tratamiento de agua adecuados no es una garantía absoluta para evitar la exposición al aluminio, ya que otros componentes de las máquinas, como bombas, membranas o filtros, pueden favorecer intoxicaciones importantes ${ }^{15,16}$. Un ejemplo de este tipo de situaciones sucedió a fines de 1992 en Portugal ${ }^{17,18}$. Durante ese año las lluvias en el sur del país fueron muy escasas, con lo cual disminuyeron los depósitos de agua y aumentaron sus partículas en suspensión. Las autoridades municipales decidieron, sin comunicarlo a los centros de hemodiálisis, incrementar la cantidad de sulfato de aluminio utilizado como floculante. El pasaje del agua contaminada a través del sistema de purificación de los centros de hemodiálisis, causó una obstrucción en los filtros y un mal funcionamiento en las membranas de ósmosis inversa. Finalmente, el agua deficientemente tratada fue utilizada para la hemodiálisis de los pacientes. El resultado fue una intoxicación aguda con 18 muertes como consecuencia de la encefalopatía causada por este metal. Este accidente ha hecho que la sensibilización en este país con respecto al riesgo de intoxicación por aluminio sea mayor que en otros. De esta forma, la calidad de las soluciones finales de diálisis en Portugal ha sido muy buena desde el primer corte realizado en 1999. Solo en el año 2001 se observó un discreto empeoramiento, 
pero en todo caso, el porcentaje de centros con concentraciones indetectables de aluminio se mantuvo por encima del 75\% en los tres estudios (tabla 1), mientras que en España en la misma época (1999) llegó sólo al 66\%. En el año 1999 y 2004, más del 80 \% de los centros de España y Portugal tenían concentraciones de aluminio en la solución final de diálisis inferiores a $2 \mu \mathrm{g} / \mathrm{L}$ (tabla 1 ).

En resumen, la calidad de la solución de diálisis en España ha mejorado notablemente desde el año 1990 hasta 2004 y prácticamente la totalidad de los centros $(99,6 \%)$ presentan niveles de aluminio por debajo del umbral "oficial" de seguridad de $10 \mu \mathrm{g} / \mathrm{L}$; si bien como se ha comentado, este nivel de seguridad ya no es seguro. Los centros de diálisis portugueses, han mantenido una muy buena calidad de las soluciones finales de diálisis desde el año 1999 hasta la actualidad.

Una política adecuada de prevención, mediante el correcto mantenimiento de los sistemas de tratamiento de aguas y un control frecuente de la eficiencia de los mismos ayudarán a mantener y mejorar la calidad de las soluciones finales de diálisis, redundando en una sustancial mejoría para los pacientes en diálisis.

\section{AGRADECIMIENTOS}

A todos los centros de diálisis que han participado en todos los estudios. A la Sociedad Portuguesa de Nefrología, por facilitar las direcciones de los centros de diálisis portugueses. A la Fundación Renal Iñigo Álvarez de Toledo, por la financiación parcial de estos estudios. A Sandra Álvarez Muñiz por su colaboración en este trabajo.

\section{BIBLIOGRAFÍA}

1. Savory J, Wills MR: Dialysis fluids as a source of aluminum accumulation. Contrib Nephrol 38:12-23, 1984.

2. Alfrey AC: Aluminum and renal disease. Contrib Nephrol 102:110-124, 1993.

3. Elliott HL, Dryburgh F, Fell GS, Sabet S, Macdougall AI: Aluminium toxicity during regular haemodialysis. Br Med J 1:1101-1103, 1978.

4. Cannata JB, Fernández Martín JL, Gómez Alonso C, Serrano Arias $\mathrm{M}$ : Control de Oligoelementos en el líquido de diálisis: un aspecto fundamental en la calidad de la diálisis de los 90. Nefrología 10:28-32, 1990.

5. Douthat W, Acuña G, Fernández Martín JL, Serrano Arias M, González Carcedo A, Canteros A, Menéndez Fraga P, Cannata JB: Exposición al aluminio y calidad del baño de diálisis: repercusión sobre los niveles de aluminio sérico. Nefrología 14:695-700, 1994.
6. Cannata JB, Douthat W, Acuña Aguerre G, FernándezMartín JL: Aluminium toxicity: the role of prevention. Life Chem Report 11:207-213, 1994.

7. Mazzuchi N, Cannata JB: Prevención, diagnóstico y tratamiento de la intoxicación alumínica: Revisión y perspectivas. Nefrología 9:15-19, 1989.

8. Fernandez-Martin JL, Canteros A, Serrano M, GonzalezCarcedo A, Diaz-Corte C, Cannata Andia JB: Prevention of aluminium exposure through dialysis fluids. Analysis of changes in the last 8 years. Nephrol Dial Transplant 13 Suppl 3:78-81, 1998.

9. Fernández Martín JL, Cannata JB: Evolución de la concentración de aluminio en la solución final de diálisis: estudio multicéntrico en centros de diálisis españoles. Nefrología 20:342-347, 2000.

10. Fernández Martín JL, Serrano M, Olaizola I, Cases A, Cannata JB: Intoxicación alumínica en la insuficiencia renal crónica. Biseden IV trimestre, 1989.

11. Serrano M, González Carcedo A, Díaz Corte C, Cannata $\mathrm{JB}$ : Aspectos de interés sobre prevención, diagnóstico y tratamiento de la osteodistrofia renal. Biseden 1:16-21, 1997.

12. Gerstenfeld S, Rodríguez-Rebollar A, Serrano Arias M, Fernández-Martín JL, Cannata-Andía J: Aluminio en solución final de diálisis en centros portugueses. Estudio multicéntrico. Rev Port Nefrol Hipert 16:83-88, 2002.

13. Fernandez-Martin JL, Menendez-Fraga P, Canteros MA, Diaz-Lopez JB, Cannata-Andia JB: Binding of aluminium to plasma proteins: comparative effect of desferrioxamine and deferiprone (L1). Clin Chim Acta 230:137-145, 1994.

14. Grosso S, Fernández Martín JL, Gómez C, Barreto S, Díaz C, Cannata JB: Prevención, diagnóstico y tratamiento de la intoxicación alumínica en España. Encuesta multicéntrica. Nefrología 16:158-166, 1996.

15. Berend K, van der Voet G, Boer WH: Acute aluminum encephalopathy in a dialysis center caused by a cement mortar water distribution pipe. Kidney Int 59:746-753, 2001.

16. Burwen DR, Olsen SM, Bland LA, Arduino MJ, Reid MH, Jarvis WR: Epidemic aluminum intoxication in hemodialysis patients traced to use of an aluminum pump. Kidney Int 48:469-474, 1995.

17. Simoes J, Barata JD, D'Haese PC, De Broe ME: Cela n'arrive qu'aux autres (aluminium intoxication only happens in the other nephrologist's dialysis centre). Nephrol Dial Transplant 9:67-68, 1994.

18. Barata JD, D'Haese PC, Pires C, Lamberts LV, Simoes J, De Broe ME: Low-dose $(5 \mathrm{mg} / \mathrm{kg}$ ) desferrioxamine treatment in acutely aluminium-intoxicated haemodialysis patients using two drug administration schedules. Nephrol Dial Transplant 11:125-132, 1996. 